The Astrophysical Journal, 485: L37-L40, 1997 August 10

(C) 1997. The American Astronomical Society. All rights reserved. Printed in U.S.A.

\title{
KILOHERTZ QUASI-PERIODIC OSCILLATION AND ATOLL SOURCE STATES IN 4U $0614+09$
}

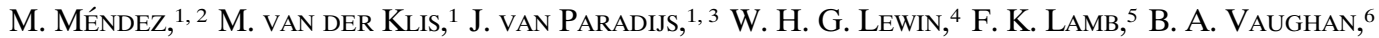 \\ E. KuUlKers, ${ }^{7}$ AND D. Psaltis ${ }^{5}$ \\ Received 1997 March 21; accepted 1997 May 30
}

\begin{abstract}
We report three $R X T E /$ PCA observations of the low-mass X-ray binary $4 \mathrm{U} 0614+09$. They show strong $(\sim 30 \% \mathrm{rms})$ band-limited noise with a cutoff frequency varying between 0.7 and $15 \mathrm{~Hz}$ in correlation with the X-ray flux, $f_{\mathrm{X}}$. We observe two nonsimultaneous $11 \%-15 \%(\mathrm{rms}) \mathrm{kHz}$ peaks near 728 and $629 \mathrm{~Hz}$ in the power spectra of two of our observations when $f_{\mathrm{X}} \sim 10^{-9} \mathrm{ergs} \mathrm{cm}^{-2} \mathrm{~s}^{-1}(2-10 \mathrm{keV})$ but find no quasi-periodic oscillations (QPOs; $<6 \% \mathrm{rms}$ ) when $f_{\mathrm{X}}$ is half that. We suggest that count rate may not be a good measure for $\dot{M}$ even in sources as intrinsically weak as 4U $0614+09$ and that QPO frequency and noise cutoff frequency track $\dot{M}$ more closely than count rate. The QPO increases in rms amplitude from $11 \% \pm 1.3 \%$ at $3 \mathrm{keV}$ to $37 \% \pm 12 \%$ at 23 $\mathrm{keV}$; the fractional amplitude of the band-limited noise is energy independent. This suggests different sites of origin for these two phenomena. The spectrum of the oscillating flux roughly corresponds to a black body with temperature $1.56 \pm 0.2 \mathrm{keV}$ and radius $500 \pm 200 \mathrm{~m}$ (other models fit as well), which might indicate that the oscillations originate at a small region on the neutron star surface.
\end{abstract}

Subject headings: accretion, accretion disks — stars: individual (4U 0614+091) — stars: neutron — X-rays: stars

\section{INTRODUCTION}

Recently, kilohertz quasi-periodic oscillations (kHz QPOs) have been discovered in 11 low-mass X-ray binaries (see van der Klis 1997 for a review). Often, the X-ray power spectra show twin $\mathrm{kHz}$ peaks moving up and down in frequency together. Sometimes a third $\mathrm{kHz}$ peak is detected near a frequency equal to the separation frequency of the twin peaks, or twice that, which suggests a beat-frequency interpretation, with the third peak near the neutron star spin frequency (or twice that). However, in Sco X-1, the twin peak separation varies, which is not consistent with a simple beat-frequency interpretation (van der Klis et al. 1997).

In the X-ray burster (Swank et al. 1978; Brandt et al. 1992) and suspected atoll source (Singh \& Apparao 1994) 4U 0614+09, twin $\mathrm{kHz}$ peaks occur (Ford et al. 1997). The peaks move between $480-800 \mathrm{~Hz}$ and $520-1150 \mathrm{~Hz}$, respectively. Their separation is consistent with being constant near $323 \mathrm{~Hz}$. There has been one $3.3 \sigma$ detection of a third peak near $328 \mathrm{~Hz}$ (in the persistent emission, whereas in the three other sources where a third peak occurred, it was seen during $\mathrm{X}$-ray bursts).

It is yet unclear how the properties of the $\mathrm{kHz}$ QPO relate to the type and state of the sources in which they have been observed. In this paper we analyze new X-ray timing and spectral data on 4U 0614+09 (preliminary report in van der

1 Astronomical Institute “Anton Pannekoek," University of Amsterdam and Center for High-Energy Astrophysics, Kruislaan 403, NL-1098 SJ Amsterdam, the Netherlands.

${ }^{2}$ Facultad de Ciencias Astronómicas y Geofísicas, Universidad Nacional de La Plata, Paseo del Bosque S/N, 1900 La Plata, Argentina.

3 Department of Physics, University of Alabama in Huntsville, Huntsville, AL 35899.

${ }^{4}$ Massachusetts Institute of Technology, Center for Space Research, Room 37-627, Cambridge, MA 02139.

5 Departments of Physics and Astronomy, University of Illinois at UrbanaChampaign, Urbana, IL 61801.

${ }^{6}$ Space Radiation Laboratory, California Institute of Technology, MC 220-47, Pasadena, CA 91125.

7 Department of Astrophysics, University of Oxford, Nuclear and Astrophysics Laboratory, Keble Road, Oxford OX1 3RH, UK.
Klis et al. 1996). We point out a number of correlations between the QPO properties and those of the broadband noise and the X-ray spectra and present for the first time an analysis of the photon energy spectrum of the oscillating flux in $\mathrm{kHz}$ QPO.

\section{OBSERVATIONS}

We observed 4U 0614+09 with the proportional counter array (PCA) on board NASA's Rossi X-Ray Timing Explorer (Bradt, Rothschild, \& Swank 1993) three times, in 1995 February, March, and April (Table 1). We simultaneously collected 2-60 keV data with a time resolution of $8 \mu$ s in eight energy bands and $16 \mathrm{~s}$ in 129 bands. The background- and dead-time-corrected count rates were 235, 571, and 256 counts $\mathrm{s}^{-1}$, respectively. A change in PCA gain between March and April affected these values only slightly. In April, only three out of the five PCA detectors were active; the five detector count rate was 426 counts $\mathrm{s}^{-1}$. We observed no X-ray bursts.

We calculated power spectra of the $8 \mu$ s data and subtracted the Poisson noise and the very large event window contribution (Zhang et al. 1995; Zhang 1995). We obtained background measurements from slew and Earth occultation data and used them to renormalize all power spectra to fractional rms squared per Hertz (see van der Klis 1995) and to correct the X-ray spectra obtained from the $16 \mathrm{~s}$ data.

\section{RESULTS}

All power spectra (Fig. 1) show strong ( 30\% rms) bandlimited noise, which we fitted with a broken power law. In two cases we observed $\mathrm{kHz}$ QPOs. These we fitted with Lorentzian peaks. The fit results are listed in Table 1. The power-law break frequency varied from $0.7 \mathrm{~Hz}$ in February, to $15.4 \mathrm{~Hz}$ in March, to $6.6 \mathrm{~Hz}$ in April.

During March and April, strong QPOs were present near 727 and $629 \mathrm{~Hz}$, respectively. The QPO properties did not vary significantly within each observation. We detect no other $\mathrm{kHz}$ QPO peaks. In particular, any peaks $323 \mathrm{~Hz}$ above or below 
TABLE 1

Power and X-Ray Spectral Parameters

\begin{tabular}{|c|c|c|c|c|}
\hline & \multirow[b]{2}{*}{1996 FEB 26} & \multicolumn{2}{|c|}{1996 MAR $16^{\mathrm{a}}$} & \multirow[b]{2}{*}{1996 APR 13} \\
\hline & & Soft-Color Dip & Main & \\
\hline & \multicolumn{4}{|c|}{ Power Spectra } \\
\hline Band-limited noise rms $(\%) \ldots$. & $30.2 \pm 0.9$ & $29.4 \pm 1.6$ & $28.1 \pm 0.7$ & $35.3 \pm 1.1$ \\
\hline Break frequency $(\mathrm{Hz}) \ldots \ldots \ldots \ldots$ & $0.7 \pm 0.1$ & $15.4 \pm 1.5$ & $15.4 \pm 0.8$ & $6.6 \pm 0.5$ \\
\hline$P_{\text {break }} \operatorname{rms}(\%)^{\mathrm{b}} \ldots \ldots \ldots$ & $12.2 \pm 1.1$ & $3.0 \pm 0.2$ & $3.2 \pm 0.2$ & $4.5 \pm 0.2$ \\
\hline QPO rms $(\%)$. & $<6$ & $11.0 \pm 1.8$ & $13.9 \pm 0.7$ & $14.6 \pm 0.5$ \\
\hline Frequency $(\mathrm{Hz}) \ldots$ & $\ldots$ & $728 \pm 8$ & $719 \pm 5$ & $629 \pm 4$ \\
\hline FWHM $(\mathrm{Hz}) \ldots \ldots \ldots \ldots \ldots$ & $\ldots$ & $54 \pm 27$ & $99 \pm 14$ & $98 \pm 9$ \\
\hline Second QPO rms (\%).. & & $<4$ & $<4$ & $<6$ \\
\hline \multirow[t]{2}{*}{ 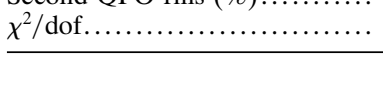 } & $284 / 229$ & $270 / 346$ & $329 / 346$ & $259 / 242$ \\
\hline & \multicolumn{4}{|c|}{ Energy Spectra } \\
\hline$N_{\mathrm{H}}\left(10^{-22} \mathrm{~cm}^{-2}\right)^{\mathrm{c}} \ldots$ & $0(<1.34)$ & $0(<0.22)$ & $0(<0.10)$ & $0.14(<0.39)$ \\
\hline$k T(\mathrm{keV}) \ldots \ldots \ldots$ & $0.42 \pm 0.05$ & & $1.10 \pm 0.18$ & \\
\hline$n$ (photon index). & $1.97 \pm 0.03$ & $2.54 \pm 0.03$ & $2.41 \pm 0.03$ & $2.30 \pm 0.03$ \\
\hline Power-law flux ${ }^{\mathrm{d}}$. & $0.36 \pm 0.02$ & $1.00 \pm 0.05$ & $0.89 \pm 0.08$ & $0.75 \pm 0.02$ \\
\hline Blackbody flux ${ }^{\mathrm{d}}$. & $0.04 \pm 0.01$ & $<0.08$ & $0.12 \pm 0.05$ & $<0.02$ \\
\hline$\chi^{2} /$ dof............. & $103 / 90$ & $92 / 94$ & $80 / 92$ & $95 / 83$ \\
\hline
\end{tabular}

Note.-Quoted errors represent $90 \%$ confidence intervals for the fits to the X-ray spectra and $1 \sigma$ confidence intervals for the fits to the power spectra. Quoted upper limits are $95 \%$ confidence. A $2 \%$ systematic uncertainty was included in the X-ray spectral errors to account for the calibration uncertainties (Cui et al. 1997).

${ }^{a}$ The March observation was divided into two parts; see text for details.

${ }^{\mathrm{b}}$ Power of the band-limited noise component at break frequency.

${ }^{\mathrm{c}} N_{\mathrm{H}}$ was consistent with zero in all of our fits. The best-fit values are given first, followed by the upper limits in parentheses.

d $2-10 \mathrm{keV}$ flux in units of $10^{-9} \mathrm{ergs}^{-2} \mathrm{~s}^{-1}$. When only an upper limit is given for the blackbody flux, this parameter was kept fixed at zero in the final fit.

our detected peaks are 3-14 times weaker than these (Table 1). The upper limit on any $328 \mathrm{~Hz}$ peak is $3.5 \%$ (rms, $95 \%$ confidence).

Count rates in March were sufficient to study the photon energy dependence of the QPO. We fitted power spectra in

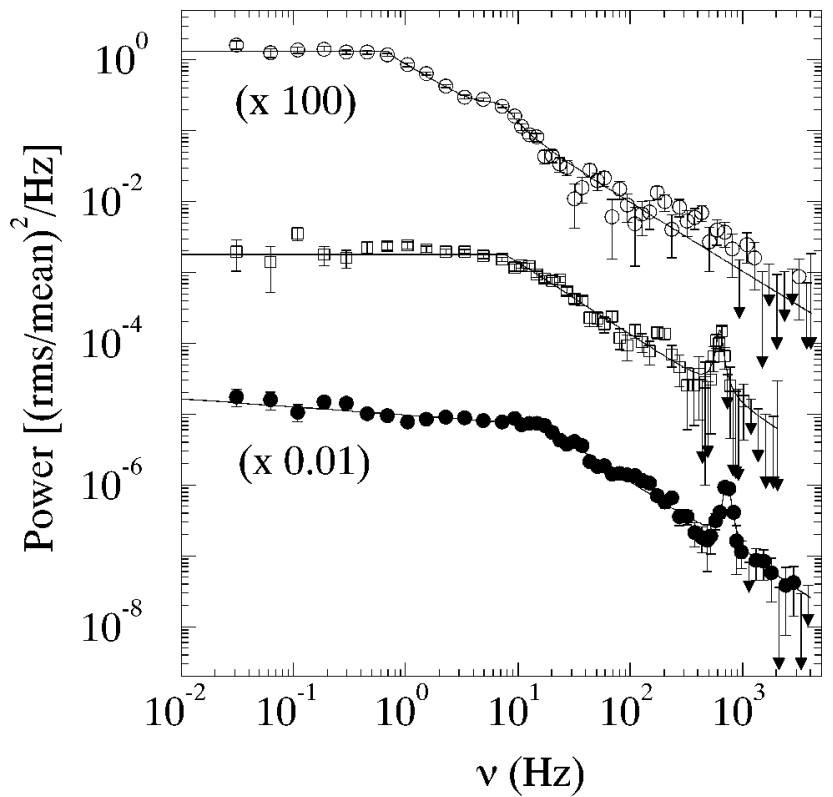

FIG. 1.-Power spectra for the three observations discussed in the paper. The data from February, March, and April are represented by open circles, filled circles, and squares, respectively. The significance of the peak at $\sim 200 \mathrm{~Hz}$ in the power spectrum from April is less than $3 \sigma$ and was not fitted. We included a QPO at $6.5 \mathrm{~Hz}$ in fitting the February spectrum. five energy bands (1.1-4.3-6.8-8.4-15.5-69.8 keV) with the break frequency, the power-law slopes, and QPO frequency and width fixed to the $2-60 \mathrm{keV}$ values (none of these varied significantly with photon energy). As shown in Figure 2 (top panel), the rms amplitude of the QPO rose from $11 \%$ at 3.3 $\mathrm{keV}$, to $20 \%$ at $11 \mathrm{keV}$, to $37 \% \pm 12 \%$ at $22.8 \mathrm{keV}$. Similar energy dependencies were seen in other sources. The fractional rms amplitude of the band-limited noise did not vary significantly as a function of photon energy.

The bottom panel of Figure 2 shows the energy spectrum of the oscillating flux (QPO rms amplitude in units of counts $\mathrm{s}^{-1}$ $\mathrm{keV}^{-1}$ vs. photon energy). For reference, we quote the results of a blackbody fit: the best fit $\left(\chi^{2}=14.4\right.$ with 3 degrees of freedom [dof]) has a temperature of $1.56 \pm 0.2 \mathrm{keV}$ and a radius of $500 \pm 200 \mathrm{~m}$ (at $3 \mathrm{kpc}$, Brandt et al. 1992). The resolution of the spectrum is low, and many spectral models are consistent with it. For example, the data can be fitted by a temperature variation of $\sim 2.5 \%$ in a blackbody spectrum of $\sim 1.1 \mathrm{keV}$ with a radius of $10 \mathrm{~km}$, or $\left(\chi^{2}=4.9\right.$ with $\left.4 \mathrm{dof}\right)$ by optical depth variations of $\sim 5 \%$ in an unsaturated Comptonization spectrum. (This latter result was obtained by fitting the fractional rms spectrum.)

To convert count rates to fluxes, we fitted the $2-50 \mathrm{keV}$ energy spectra with a black body plus a power law modified by interstellar absorption (Table 1). In April and during a dip in soft color in March (below), a power law alone could fit the spectrum. The inferred $2-10 \mathrm{keV}$ fluxes were $0.4,1.0$, and $0.75 \times 10^{-9}$ ergs $\mathrm{cm}^{-2} \mathrm{~s}^{-1}$ in February, March, and April, respectively; the $2-50 \mathrm{keV}$ fluxes are $50 \%, 30 \%$, and $40 \%$ higher, respectively. As $N_{\mathrm{H}}$ is low, absorbed and "unabsorbed" fluxes are the same. We tried various other spectral shapes, 


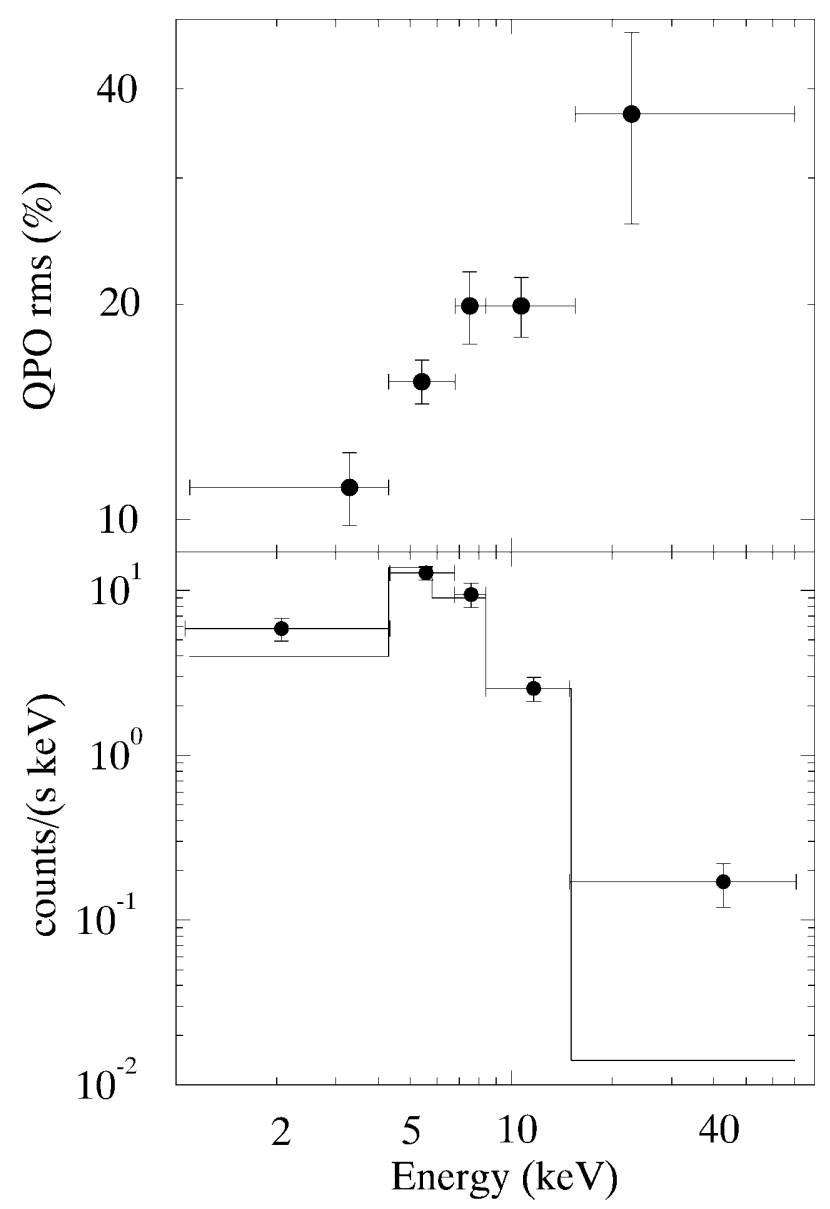

FIG. 2.-Top, Fractional rms amplitude vs. photon energy spectrum of the QPO from 1996 March; bottom, energy spectrum of the oscillating flux, with blackbody fit, for the same data set.

with no effect on the derived fluxes. Count rate, flux, and power spectra were not affected by the soft-color dip.

To compare 4U 0614+09 with confirmed atoll sources (see Hasinger \& van der Klis 1989), we produced an X-ray color-color diagram using the three spectral bands $2-5-10-50$ $\mathrm{keV}$ (Fig. 3). We corrected for detector gain changes by comparing the count rate ratios with incident flux ratios

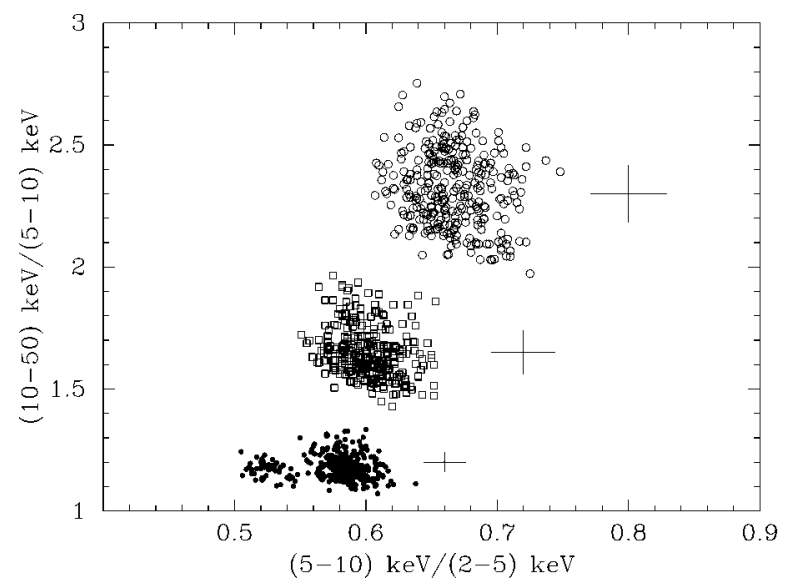

FIG. 3.-Color-color diagram showing all the observations mentioned in the paper. Symbols are the same as in Fig. 1. Each point represents $16 \mathrm{~s}$ of data. Typical error bars for each observation are indicated. obtained in the same bands from the spectral fits. Once again we tried different spectral shapes and found negligible effects on the color corrections. There was a dip of $\sim 800 \mathrm{~s}$ in soft color starting $5200 \mathrm{~s}$ into the March observation. Apart from this, the colors within each observation show no significant changes, with upper limits on any intrinsic color variations of $\sim 10 \%$. Except for the March excursion, this is entirely consistent with atoll-source island-state behavior.

\section{DISCUSSION}

From color-color diagrams, Singh \& Apparao (1994) suggested 4U 0614+09 to be an atoll source. Our colorcolor diagrams, spectral fits, and simultaneous strong bandlimited noise with $0.7-15 \mathrm{~Hz}$ cutoff frequencies confirm that the correlated X-ray spectral and timing properties of $4 \mathrm{U} 0614+09$ are those of an atoll source, in the island state during our observations. The large fractional amplitude and low cutoff frequency of the band-limited noise and the relatively hard X-ray spectra make the source similar to the atoll sources $4 \mathrm{U} 1608-52$ and $4 \mathrm{U} 1705-44$ in the island state (Hasinger \& van der Klis 1989; Langmeier, Hasinger, \& Trümper 1989; Yoshida et al. 1993; Berger \& van der Klis 1997), which in turn resemble the black hole candidates Cyg X-1 and GX 339-4 in the low and intermediate states (van der Klis 1994a; Berger \& van der Klis 1997; Belloni et al. 1996; Crary et al. 1996; Méndez \& van der Klis 1997). All these sources are quite hard. Their energy spectra fit a soft component with $k T \sim 1 \mathrm{keV}$ plus a power law with a photon index of 1.6-2.5. Their power spectra show band-limited noise with a $\sim 0.1-10 \mathrm{~Hz}$ cutoff frequency that is anticorrelated with the level of the flat top (Belloni \& Hasinger 1990; Méndez \& van der Klis 1997). This anticorrelation holds also for our power spectra of $4 \mathrm{U} 0614+09$. Break frequencies and fractional rms at the break are fully consistent with the existing relation between these quantities from other sources (see Fig. 3 of Méndez \& van der Klis 1997).

If, as proposed (van der Klis 1994b; Méndez \& van der Klis 1997), the break frequency of the band-limited noise component here is an indication for $\dot{M}$, then $\dot{M}$ increased from February to March and then decreased to an intermediate value in April. This is consistent with the variations in X-ray flux among our three observations. Note that this is not always the case in similar sources (e.g., 4U 1608-52; Yoshida et al. 1993).

In this picture, we observe no $\mathrm{kHz}$ QPO ( $<6 \% \mathrm{rms})$, when $\dot{M}$ is lowest, and $11 \%-15 \%$ amplitude QPOs at higher inferred $\dot{M}$ values. Although $\mathrm{kHz}$ QPOs often get stronger when inferred $\dot{M}$ drops (in 4U $1636-53$, Wijnands et al. 1997; KS 1731-260, Wijnands \& van der Klis 1997; 4U 1820-30, Smale, Zhang, \& White 1997), apparently in 4U 0614+09 there is a value of $\dot{M}$ below which the QPO becomes weaker.

We now turn to the identification of our QPO peaks. In our data, at most one peak is present at each time, whereas for similar count rates, Ford et al. (1997) usually find twin peaks. The count rate-QPO frequency relations do not clearly identify our peaks as either the higher or the lower frequency ones (Fig. 4). The QPOs amplitudes do give a clue. Ford et al. (1997) found the lower frequency peak to have an rms amplitude $0.25-0.75$ times that of the higher frequency peak when the count rate was near 400 counts s ${ }^{-1}$ and $0.5-1.5$ that at $600-700$ counts $\mathrm{s}^{-1}$. If our peaks are the higher frequency ones, these ratios are less than 0.3-0.4 in our data. If they are 


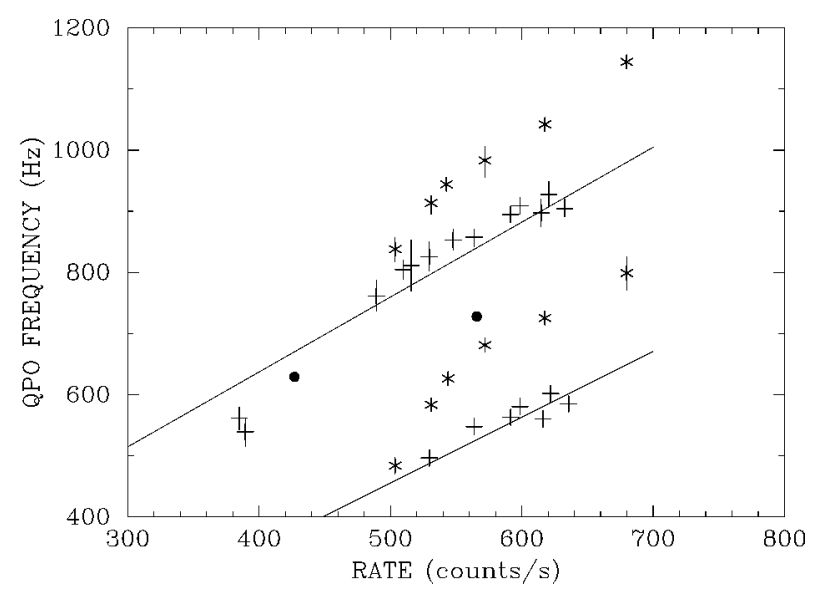

FIG. 4.-The count rate vs. QPO frequency diagram of all $4 \mathrm{U} 0614+09$ data reported to date. Pluses, asterisks, and power-law relations have been taken from Ford et al. (1997); filled circles represent the new data presented in this paper.

the lower frequency ones, these values are greater than 2.4-3.5. As our count rates are 400-600 counts s ${ }^{-1}$, our peaks are probably the higher frequency ones. Then, in our data we have a positive correlation between QPO frequency and count rate, but one that does not fit either of the relations observed previously (see Fig. 4).

The QPO frequency versus count rate diagram of $4 \mathrm{U} 0614+09$ (Fig. 4) shows that there is no single relation that describes the correlation between those two quantities. This means that either count rate or frequency (or both) does not track $\dot{M}$ well. We suggest that count rate is not a good measure of $\dot{M}$. Both spectral changes and variations in the anisotropy of the emission can destroy the expected correspondence between these two quantities. To the extent that bolometric corrections can be accurately performed, a conversion to energy flux adjusts for the spectral changes. However, the experience from the $Z$ sources (at admittedly much higher $\dot{M}$ values) shows that even derived bolometric X-ray fluxes do not always track $\dot{M}$ well. In $4 \mathrm{U} 1608-52$, in a very similar series of island-state observations as the present ones of $4 \mathrm{U} 0614+09$, Yoshida et al. (1993) found that the bolometric flux did not exhibit a one-to-one correspondence to cutoff frequency of the band-limited noise. Whereas there are many processes that can quite easily change the observed X-ray count rates, colors, spectral parameters, and even bolometric fluxes from their original values, the noise cutoff frequency is not so easily affected. The noise cutoff can, in principle, be lowered by scattering delays. The $\mathrm{kHz}$ QPO frequency, on the other hand, is very likely a direct diagnostic of the dynamics of the inner flow and is therefore very hard to modify by any propagation effect. It is possible, then, that QPO frequency is well correlated to $\dot{M}$ but that count rate is not. It will be of great interest to see whether the correlation suggested by the joint decrease of the noise cutoff frequency and QPO frequency from March to April (see Table 1) will hold up in future analyses of other data or whether a QPO frequency-flux relation will turn out to be the more reproducible, as this may provide clues to both the best measure of $\dot{M}$ in atoll sources in extreme island states (and perhaps black hole candidates) and to the physical origin of $\mathrm{kHz}$ QPO and broadband noise in these systems.

We observed a very strong energy dependence in the $\mathrm{kHz}$ QPO but no energy dependence in the band-limited noise. One plausible interpretation of this-namely, that the lowenergy photons undergo scattering with a characteristic delay timescale intermediate between the QPO timescale $(1 \mathrm{~ms})$ and that of the noise $(0.1-10 \mathrm{~s})$ - can probably be excluded on the basis of the extremely small $(20 \mu \mathrm{s}$ and $<50 \mu \mathrm{s})$ time lags between the $\mathrm{kHz}$ QPO signals at different photon energies recently reported by Vaughan et al. (1997). (Of course, scattering with shorter characteristic timescales cannot be excluded and is in fact likely on other grounds.) The $\mathrm{kHz}$ QPO spectrum, which resembles a blackbody shape with a characteristic temperature of $1.6 \mathrm{keV}$ and radius of $500 \mathrm{~m}$, might indicate an origin associated with a relatively small area on the neutron star surface, whereas the band-limited noise may have a different site of origin, perhaps in the inner disk. This would be in accordance with the observation that band-limited noise is a common trait among neutron stars and black holes (van der Klis 1994a), whereas correlated twin $\mathrm{kHz}$ QPO peaks are so far unique to neutron star systems.

This work was supported in part by the Netherlands Organization for Scientific Research (NWO) under grant PGS 78-277 and by the Netherlands Foundation for Research in Astronomy (ASTRON) under grant 781-76-017. M. M. is a fellow of the Consejo Nacional de Investigaciones Científicas y Técnicas de la República Argentina. W. H. G. L. acknowledges support from the National Aeronautics and Space Administration. J. v. P. acknowledges support from the National Aeronautics and Space Administration through contract NAG53269. F. K. L. acknowledges support from NSF, through grant AST 93-15133, and NASA, through grant 5-2925.

\section{REFERENCES}

Belloni, T., \& Hasinger, G. 1990, A\&A, 227, L33

Belloni, T., Méndez, M., van der Klis, M., Hasinger, G., Lewin, W. H. G., \& van Paradijs, J. 1996, ApJ, 472, L107

Berger, M., \& van der Klis, M. 1997, A\&A, in press

Bradt, H. V., Rothschild, R. E., \& Swank, J. 1993, A\&AS, 97, 355

Brandt, S., Castro-Tirado, A. J., Lund, N., Dremin, V., Lapshov, I., \& Sunyaev,

R. 1992, A\&A, 262, L15

Crary, D. J., et al. 1996, ApJ, 462, L71

Cui, W., Heindl, W. A., Rothschild, R. E., Zhang, S. N., Jahoda, K., \& Focke, W. 1997, ApJ, 474, L57

Ford, E., et al. 1997, ApJ, 475, L123

Hasinger, G., \& van der Klis, M. 1989, A\&A, 225, 79

Langmeier, A., Hasinger, G., \& Trümper, J. 1989, ApJ, 340, L21

Méndez, M., \& van der Klis, M. 1997, ApJ, 479, 926

Singh, K. P., \& Apparao, K. M. V. 1994, ApJ, 431, 826

Smale, A. P., Zhang, W., \& White, N. E. 1997, IAU Circ. 6507

Swank, J. H., Becker, R. H., Boldt, E. A., Holt, S. S., \& Serlemitsos, P. J. 1978, MNRAS, 182,349 van der Klis, M. 1997, in the Proc. Wise Obs. 25th Anniversary Symp. Astron.

Time Ser., Tel Aviv, in press

. 1995, in X-Ray Binaries, ed. W. H. G. Lewin, J. van Paradijs, \&

E. P. J. van den Heuvel (Cambridge: Cambridge Univ. Press), 252 1994a, ApJS, 92, 511

1994b, A\&A, 283, 469

van der Klis, M., van Paradijs, J., Lewin, W. H. G., Lamb, F. K., Vaughan, B. A., Kuulkers, E., \& Augusteijn, T. 1996, IAU Circ. 6428

van der Klis, M., Wijnands, R. A. D., Horne, K., \& Chen, W. 1997, ApJ, 481, L97

Vaughan, B. A., et al. 1997, ApJ, 483, L115

Wijnands, R. A. D., \& van der Klis, M. 1997, ApJ, 482, L65

Wijnands, R. A. D., van der Klis, M., van Paradijs, J., Lewin, W. H. G., Lamb, F. K., Vaughan, B. A., \& Kuulkers, E. 1997, ApJ, 479, L141

Yoshida, K., Mitsuda, K., Ebisawa, K., Ueda, Y., Fujimoto, R., Yaqoob, T., \& Done, C. 1993, PASJ, 45, 605

Zhang, W. 1995, XTE/PCA Internal Memo, 5-23-95

Zhang, W., Jahoda, K., Swank, J. H., Morgan, E. H., \& Giles, A. B. 1995, ApJ, 449,930 